\title{
FDA approves Galafold, a triumph for Amicus
}

Following accelerated US Food and Drug Administration approval in August of Amicus Therapeutics's first product, Galafold (migalastat), for treating some types of the progressive $\mathrm{X}$-linked lysosomal storage disorder Fabry disease, it is now time for a head-to-head comparison of the oral, small molecule drug against standard-of-care intravenously administered enzyme replacement therapy. John Crowley, CEO of Amicus, is wont to speak of "a duty to obsolete our own technology" in the company's pursuit of cures for rare diseases, but the more immediate question is whether Galafold can replace enzyme replacement therapy.

In terms of the therapeutic index of the two modalities, the 60-patient phase 3 ATTRACT study of Galafold demonstrated a similar effect on the primary endpoint of kidney function to the two marketed enzyme replacement therapies: Sanofi's Fabrazyme (agalsidase beta) and Shire's Replagal (agalsidase alfa)

In an earlier Galafold phase 3 study in 67 patients, the FACETS trial, treatment-naive patients receiving the Amicus small molecule for 12 months had durable reductions in levels of kidney globotriaosylceramide (GL-3), a lipid that accumulates to toxic levels in Fabry disease.

Meanwhile, placebo patients in the FACETS trial who switched to Galafold also had a statistically significant reduction in GL-3 after a year. Patient questionnaires showed a statistically significant improvement in diarrhea and indigestion. But pain severity scores did not change from baseline to the end of the study in either ATTRACT or FACETS.

Fabry disease is caused by a mutation in the GLA gene that gives rise to a deficiency or dysfunction in the lysosomal $\alpha$-galactosidase ( $a-G a l-A)$ enzyme. This enzyme is required to metabolize GL-3 and plasma globotriaosylsphingosine (lyso-Gb3). When these glycosphingolipids accumulate, they cause damage in tissues throughout the body.

Galafold acts by reversibly binding to the active site of $\alpha$-Gal-A, stabilizing specific

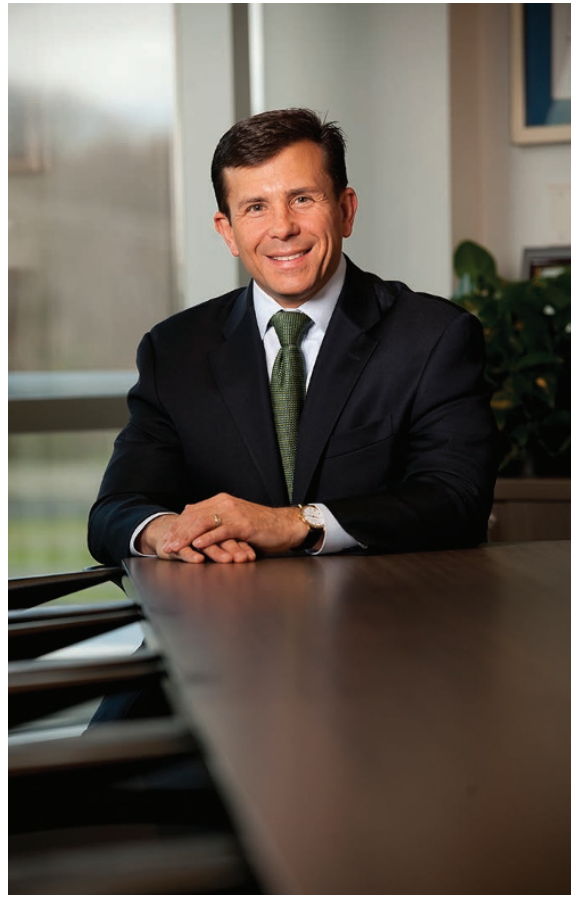

John Crowley, chairman and CEO of Amicus Therapeutics, started the company to find a treatment for a daughter who is afflicted by Pompe disease, a rare lysosomal storage disorder.

mutant forms, and chaperoning the enzyme to lysosomes, where dissociation of Galafold allows $\alpha$-Gal-A to catabolize GL-3 and lysoGb3 deposits.

Amicus has set up a pharmacogenetics website and developed a diagnostic to test whether patients have a mutation that is amenable to treatment with Galafold. There are 841 mutations of the GLA gene identified as causing Fabry disease, of which 348 are known to be amenable. This represents "a great advance in personalized medicine and regulatory science," Crowley said when commenting on the approval.

Raphael Schiffmann of Baylor Scott \& White Research Institute, Dallas, treated the first patient ever to receive Galafold in a clinical trial. "Many years later, I am pleased that more patients will have access to this precision medicine," he said, commenting on the approval. Jack Johnson of the Fabry Support and Information Group agreed. "Amicus has provided a much needed new treatment option for many Fabry patients."

For those patients who respond to Galafold, there are tantalizing hints that it could be more effective than enzyme replacement therapy. But for now, the advantages lie in the better quality of life and greater convenience of taking a 123-mg pill every two days, versus infusions every two weeks.

That was certainly the verdict of the Canadian Fabry Association in its submission to the Canadian Agency for Drugs and Technologies in Health. Patients said infusions are "highly disruptive to daily living." Eight of 90 patients who took part in the association's survey had switched from enzyme replacement therapy to Galafold. All cited convenience of administration and better symptom management as benefits.

Amicus also has given itself an edge-at least initially_in pricing. "We price our medicines at or below similar competitor products," says Crowley. "By pricing below parity to current standard-of-care enzyme replacement therapy, we deliver the value of a precision medicine option for patients with amenable GLA variants without the infusion-associated costs that come with enzyme replacement therapy," he says.

A phase 4 study mandated by FDA and other in-market data will in time show whether Galafold is therapeutically superior to currently marketed enzyme replacement. However, that will not address the needs of patients who do not have amenable mutations. But combining Galafold with a next-generation enzyme replacement therapy, a product Amicus has in development, could indeed render enzyme replacement monotherapy obsolete.

Nuala Moran London

\footnotetext{
66 "Given that this flu virus is a potential threat to humanity, not sharing it immediately with the global network of WHO laboratories ... is scandalous. Many could die needlessly if China denies international access to samples." Andrew C. Weber, former Pentagon biological defense expert, reacts to Chinese health authorities not releasing H7N9 specimens. (The New York Times, 27 August 2018)
}

"When I started out being excited by science-but seeing that there weren't any women scientists-I thought I had no prospects whatsoever. The one thing that I really wanted was to have the respect of my peers for the scientific contributions I made, and for my participation in the scientific community." Joan Steitz, recipient of the 2018 Lasker-Koshland Award for Special Achievement in Medical Science, on being recognized for her work on RNA, but also for championing the work of women scientists. (The New York Times, 11 September 2018)

\footnotetext{
"Nearly everyone I know is putting the words 'Alzheimer's disease' in their grants in an effort to tap into the money." Matt Kaeberlein, University of Washington, Seattle, remarks on how NIH efforts to turn billions of new funding into treatment is influencing researchers' applications. Science, 30 August 2018)
} 